\title{
MOLECULAR CHARACTERIZATION OF SARCOCYTIS FUSIFORMIS AND SARCOCYSTIS BUFFALONIS INFECTING WATER BUFFALOES (BUBALUS BUBALIS) FROM EGYPT
}

\author{
${ }^{1}$ Mahmoud El-Seify, ${ }^{2,6}$ Ahmed El-Morsey, ${ }^{3}$ Mosaad Hilali, ${ }^{2}$ Ahmed Zayed \\ ${ }^{4,6}$ Khaled El-Dakhly, ${ }^{5,6}$ Mohie Haridy, ${ }^{6}$ Hiroki Sakai and ${ }^{6}$ Tokuma Yanai \\ ${ }^{1}$ Department of Parasitology, Faculty of Veterinary Medicine, KafrElsheikh University, Egypt \\ ${ }^{2}$ Department of Parasitology and Animal Diseases, Veterinary Research Division, \\ National Research Center, Dokki, El-Behooth St, 12622, Giza, Egypt \\ ${ }^{3}$ Department of Parasitology, Faculty of Veterinary Medicine, Cairo University, Egypt \\ ${ }^{4}$ Department of Parasitology, Faculty of Veterinary Medicine, \\ Beni-Suef University, Beni-Suef 62511, Egypt \\ ${ }^{5}$ Department of Pathology and Clinical Pathology, Faculty of Veterinary Medicine, \\ South Valley University, Qena 83523, Egypt \\ ${ }^{6}$ Laboratory of Veterinary Pathology, Faculty of Applied Biological Sciences, \\ Gifu University, 1-1 Yanagido, Gifu 501-1193, Japan
}

Received 2014-04-10; Revised 2014-04-14; Accepted 2014-04-16

\begin{abstract}
Parasites of the genus Sarcocystis have the ability to infect wide range of animals, birds and reptiles. Water buffaloes are intermediate hosts for four Sarcocystis species; S. fusiformis, S. buffalonis, S. levinei and $S$. dubeyi. There was no previous molecular identification of Sarcocystis spp. parasitizing water buffaloes from Egypt. The current study was established to screen infection rates and apply genetic identification of the macroscopic Sarcocystis spp. infecting Egyptian water buffalos in El-Gharbia province, Egypt. Sarcocysts of both $S$. fusiformis and $S$. buffalonis were grossly examined through meat inspection in slaughterhouse, followed by histological identification of the detected sarcocysts. Finally, molecular identification using PCR, sequencing and phylogenetic analysis of the 18S rRNA gene was conducted. Comparison of the obtained sequences of the local tested isolates of both $S$. fusiformis and S. buffalonis with those previously reported for different Sarcocystis species those were recorded in Genbank, revealed that all the tested specimens represented both S. fusiformis and S. buffalonis. Moreover, sequence alignments of the partial $18 \mathrm{~S}$ rRNA gene ascertained the presence of genetic variations revealing the heterogenic nature of the gene among different strains of the same Sarcocystis species in relation to the geographic distribution.
\end{abstract}

Keywords: Sarcocyst, 18S rRNA, Phylogeny, Water Buffalo, Egypt

\section{INTRODUCTION}

Sarcocystis is a large genus of intracellular, cystforming coccidian parasites belonging to the protozoa (Sporozoa: Apicomplexa) with an obligate two host life cycle between predators as final hosts and their prey animals as intermediate hosts. Sarcocystis spp.
Are highly prevalent in livestock and are considered to be host specific (Dubey et al., 1989).

Few Sarcocystis species are pathogenic to animals with a great economic impact as they cause reduced weight gain, poor feeding efficiency, anorexia, fever, anemia, muscle weakness, reduced milk yield, abortion and mortality of intermediate hosts such as $S$. cruzi in cattle, S. tenella in

Corresponding Author: Tokuma Yanai, Laboratory of Veterinary Pathology, Faculty of Applied Biological Sciences, Gifu University, 1-1 Yanagido, Gifu 501-1193, Japan Tel/Fax: +81-58-293-2943 
sheep, S. capricanis in goats and S. miescheriana in swine (Dubey et al., 1989; El-Morsey, 2010; El-Morsey et al., 2014). Macroscopic visible cysts, like $S$. fusiformis, $S$. buffalonis and $S$. gigantea, also may render meat unaesthetic and thus lead to condemnation of infected carcasses (Dubey et al., 1989; Yang et al., 2000; Hilali et al., 2011). To date, four characterized species of Sarcocystis have been reported and described in the water buffalo as an intermediate host. S. fusiformis and Sarcocystis buffalonis form macroscopic sarcocysts with cats as the definitive host, while Sarcocystis levinei and Sarcocystis dubeyi form microscopic sarcocysts. Despite dogs are known to be the definitive hosts for S. levinei, the definitive host (s) for $S$. dubeyi has not yet been identified (Huong, 1999a; 1999b; Huong and Uggla, 1999; Huong et al., 1997; Hilali et al., 2011).

The present work aimed to conduct prevalence and molecular identification of the macroscopic Sarcocystis species infecting Egyptian water buffalos in El-Gharbia province, Egypt, using gross identification by meat inspection, histological examination and molecular identification of the revealed Sarcocystis species using PCR, sequencing and phylogenetic analysis of (the small ribosomal subunit) $18 \mathrm{~S}$ rRNA gene.

\section{MATERIALS AND METHODS}

\subsection{Collection of Samples}

Specimens were obtained from 528 water buffaloes slaughtered at the municipal slaughterhouse in ElMahalla city, El-Gharbia province (coordinates: $30.881^{\circ} \mathrm{N} 31.06^{\circ} \mathrm{E}$ ), Egypt during the period from December 2011 to November 2012. According to age, buffaloes were categorized into 2 groups, the first from 2-3 years and the second included animals of more than 5 years. Age of examined animals was determined based on the visual examination of both horns and teeth.

\subsection{Gross Examination and Histopathology}

Oesophageal muscles were examined by the naked eye to detect macroscopic sarcocysts. Macroscopic visible sarcocysts were carefully isolated from muscle samples and fixed in $10 \%$ formalin and routinely processed for histopathologic examination, sectioned at $5 \mu \mathrm{m}$ and examined at magnifications of $40 \times, 100 \times, 400 \times$ and $1000 \times$.

\subsection{Molecular Identification}

Prior to molecular confirmation, species identification was done on freshly collected muscle samples containing cysts of both species, using light microscopy according to (Dubey et al., 1989; Odening et al., 1995; 1996). Fresh sarcocysts of the two identified species were isolated from muscle tissues with sterile brand needles for each cyst, washed 3-4 times with $0.9 \% \mathrm{NaCl}$ as previously described according to (Yang et al., 2001b). Five macroscopic sarcocysts were obtained from each of the identified Sarcocystis species. Samples were incubated into phosphate buffer saline (pH 7.2) and transferred as soon as possible to the Biotechnology Center, Faculty of Veterinary Medicine, Cairo University for further processing.

\subsection{DNA Extraction}

DNA was extracted from sarcocysts obtained from both isolates identified as $S$. fusiformis and $S$. buffalonis using Qiagen DNeasy Blood and Tissue kit ${ }^{\circledR}$ according to manufacturer protocol.

\subsection{PCR Amplification}

PCR reactions were applied for five macrocysts from both isolates of $S$. fusiformis and $S$. buffalonis using the primer set: Forward (5CGCCCTTTTAGTGAGGGTGT3') and reverse (5 TACGAATGCCCCCAACTGTC 3') those were designed according to (18S rRNA sequence of $S$. fusiformis accession number U03071), using the primer BLAST tool on the NCBI of the Genbank (http://www.ncbi.nlm.nih.gov/tools/primer-

blast/primertool.cgi), to target the 18S rRNA gene in a final reaction volume of $50 \mu \mathrm{L}$ containing $1 \mu \mathrm{L}$ DNA template (100 ng), $0.5 \mu \mathrm{L}$ (50 pmol) of each primer, $2 \mu \mathrm{L}$ dNTPs (Advanced Bio-enzymes, UK), $1 \mu \mathrm{L}$ DNA polymerase (2 $\mathrm{U})$ (Advanced Bio-enzymes, UK), $5 \mu \mathrm{L}$ of $10 \times$ buffer $(500$ $\mathrm{mM} \mathrm{KCl}, 100 \mathrm{mM}$ Tris $\mathrm{HCl} \mathrm{pH} 9.0,1.5 \mathrm{mM} \mathrm{MgCl}_{2}$ ) and $40 \mu \mathrm{L}$ Distilled water. Initial denaturation at $94^{\circ} \mathrm{C}$ for 4 min, 40 cycles of denaturation at $94^{\circ} \mathrm{C}$ for $1 \mathrm{~min}$, annealing at $55^{\circ} \mathrm{C}$ for $1 \mathrm{~min}$ and extension at $72^{\circ} \mathrm{C}$ for $90 \mathrm{sec}$ and then final extension at $72^{\circ} \mathrm{C}$ for $1 \mathrm{~min}$. The amplification reactions were carried out in PCR Thermocycler (PTC 100 MJR Research, USA). Two $\mu \mathrm{L}$ of bromophenol blue were added to the aliquots of PCR products and the corresponding amplicons were electrophoresed on $2.5 \%$ agarose gel, stained with ethidium bromide, examined using a UV transilluminator and photographed.

\subsection{DNA Sequencing, Sequence Alignment and phylogenic Analysis}

PCR amplicons were purified and prepared for sequencing using Qiaquick gel extraction kits ${ }^{\circledR}$ according to manufacturer recommendations. The sequence of the clones was determined from forward direction (5'-3'). Multiple DNA sequence alignments were carried out for 2 PCR products, one from each species. The $18 \mathrm{~S}$ rRNA gene fragments were sequenced using the same primers used in PCR. Samples were analyzed on an ABI prism 
377 capillary DNA sequencer. The sequence alignments were extensively checked by eye to determine the percentages of similarities and discrepancies. Initially, comparison was done between the sequences of the local tested isolates of $S$. fusiformis and S. buffalonis, then between the sequence alignments of the two species with those of previously published species of $S$. fusiformis AF179627, AF179626, (Yang et al., 2001b) and U03071, (Holmdahl et al., 1994) and S. buffalonis AF 017121, (Holmdahl et al., 1999) to determine the percentages of similarities and variability among them. To reconstruct the phylogenetic relationship, $421 \mathrm{bp}$ of the $18 \mathrm{~S}$ rRNA gene representing each of PCR products of the two isolates (accessions numbers JQ821390-JQ821391) were included in a comparative analysis with those previously published in the NCBI using BLAST (http://blast.ncbi.nlm.nih.gov/), on the basis of highly similar sequences available on Genbank data base and by means of maximum parsimony trees reconstructed from Kimura 2-parameter distances from 1,000 bootstrap replicates of multiple sequence alignments created using CLUSTALW, as implemented by MEGA version 4 (Tamura et al., 2007) The Neospora caninum (GQ 899206) was employed as outgroup.

\section{RESULTS}

\subsection{Prevalence study}

The present investigation revealed higher infection rates with both Sarcocystis species in old-aged animals ( 5 years and above), than those occurred in younger animals (2-3 years). Among old-aged animals, 68.2\% (253/371) and $13.2 \%$ (49/371) were found to be infected with $S$. fusiformis and $S$. buffalonis, respectively. Infection rates in younger animals were 17.2\% (27/157) and $10.2 \%$ (16/157). Mixed infections with both species were found in $83.8 \%(311 / 371)$ and $29.9 \%$ (47/157) for S. fusiformis and S. buffalonis, respectively (Table 1).

\subsection{Morphologic Identification}

S. fusiformis cysts were macroscopic, opaque white in color, spindle shaped, measured $0.5-3.0 \times 0.3-0.7 \mathrm{~cm}$ and located sometimes under the serosal sheet or deeply situated within the esophageal musculature (Fig.1).

Double opposing arrow heads refer to the true cyst wall, single arrows refer to the nuclei of the muscle fiber layer adherent to the cyst wall, S; septa, M: peripheral metrocytes, B; bradyzoites (located toward the center of the cyst). Bar $=10$ microns ( $\mathrm{H}$ and Estain).

Histologically; the cyst was surrounded by smooth, thin cyst wall $(1-3 \mu \mathrm{m})$. Fine Septa $(\mathrm{S})$ originated from the inner layer dividing the cavity of the cyst into several compartments of different sizes, those were packed with faintly-stained Metrocytes (M) in the periphery and Bradyzoites (B) toward the center (Fig. 2).

On the other hand, S. buffalonis cysts were visible to the naked eye as white threads running parallel to the longitudinal axis of the muscle fibers; often immediately under the serosal sheet of the esophageal muscles. They were 1-3×0.1-0.5 mm $(\mathrm{n}=50)$. In few cases, cysts of $S$. buffalonis might appear twisted due to the postmortum tension of the underlying muscle fibers, (Fig. 3) and surrounded by a striated thick cyst wall measured 4-6 $\mu \mathrm{m}$ $(n=25)$ (Fig. 4). The internal structure was similar to that of $S$. fusiformis.

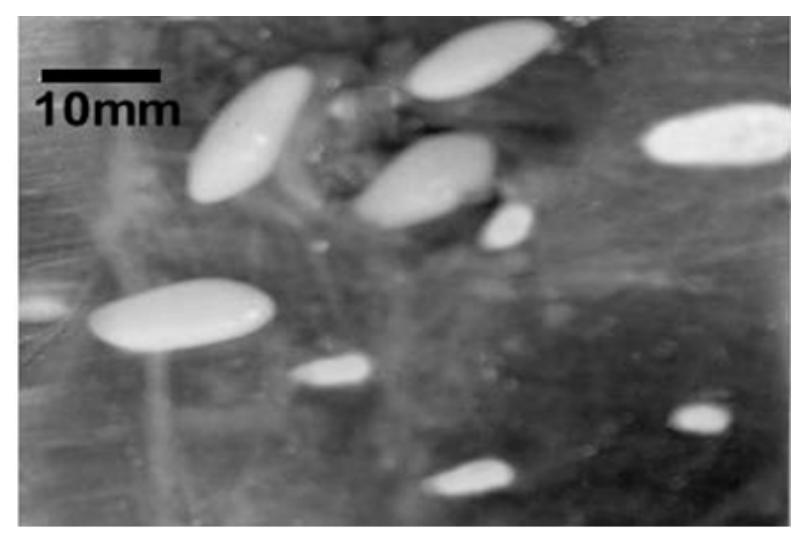

Fig. 1. Macroscopic S. fusiformis cysts in the oesophageal muscle of water buffalo. Bar $=10 \mathrm{~mm}$

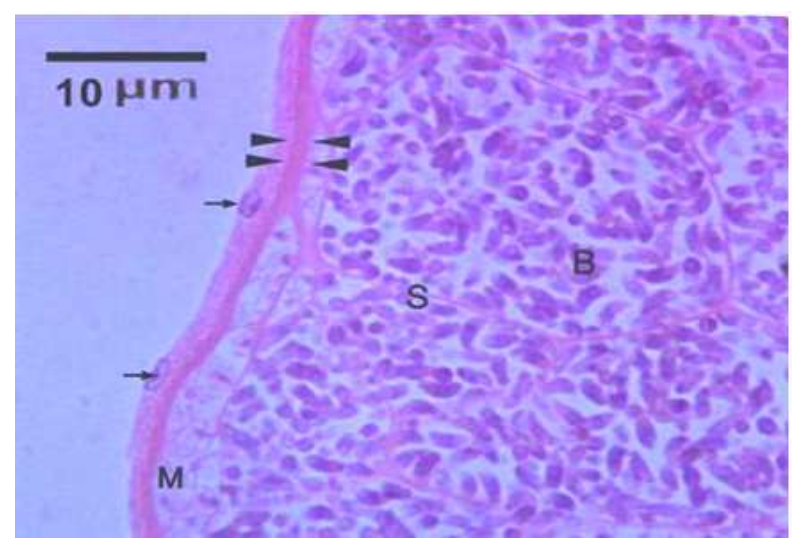

Fig. 2. Histological section of a mature $S$. fusiformis cyst showing the basic elements of the sarcocyst: Double opposing arrow heads refer to the true cyst wall, single arrows refer to the nuclei of the muscle fiber layer adherent to the cyst wall, S; septa, M: Peripheral metrocytes, B; bradyzoites (located toward the center of the cyst). Bar $=10$ microns. $(\mathrm{H}$ and Estain) 


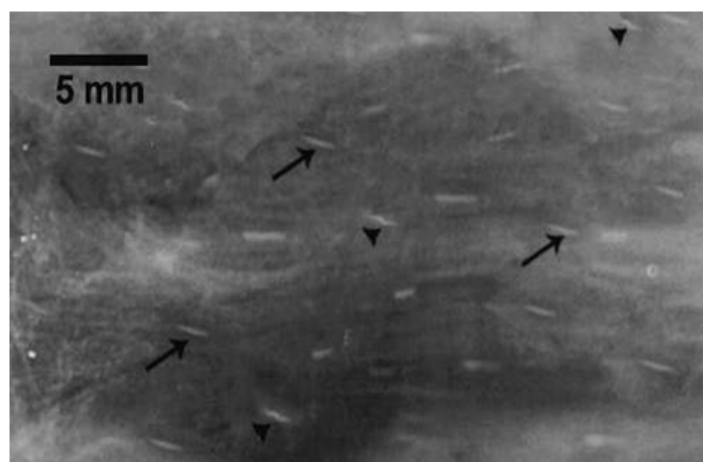

Fig. 3. S. buffalonis in the esophageal muscle of water buffalo showing thread like cysts in the oesophagus of water buffalo (arrows), some times appear twisted due to post mortum tension of the muscles (arrow heads). Bar $=5 \mathrm{~mm}$

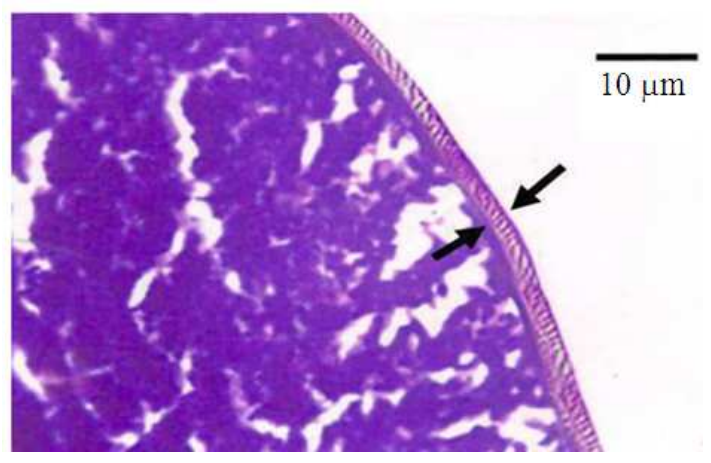

Fig. 4. S. buffalonis: Histological section of the cyst from the esophageal muscle of water buffalo, showing cross section of the cyst showing striated (palisade like) thick cyst wall (opposing arrows). (H\&E) stain. Bar $=10$ microns

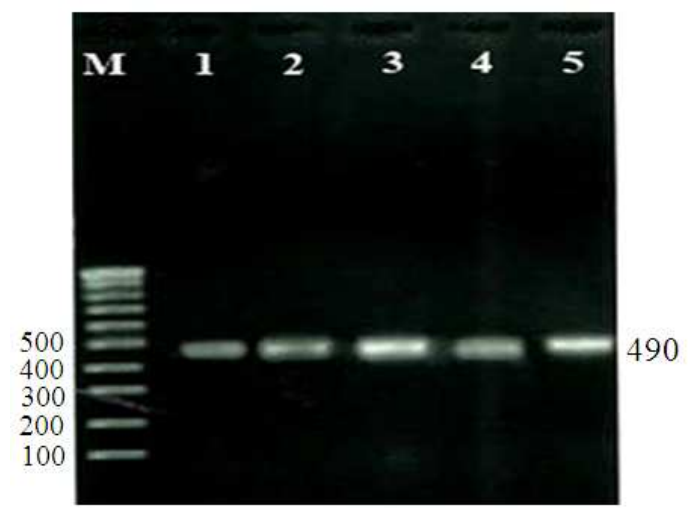

Fig. 5. Electrophoresis of the $18 \mathrm{~S}$ rRNA gene used for PCR assay for the local tested isolates of $S$. fusiformis. Lane M 100-bp ladder = DNA marker. Lanes 1-5 represent the positive diagnostic bands of the tested specimens

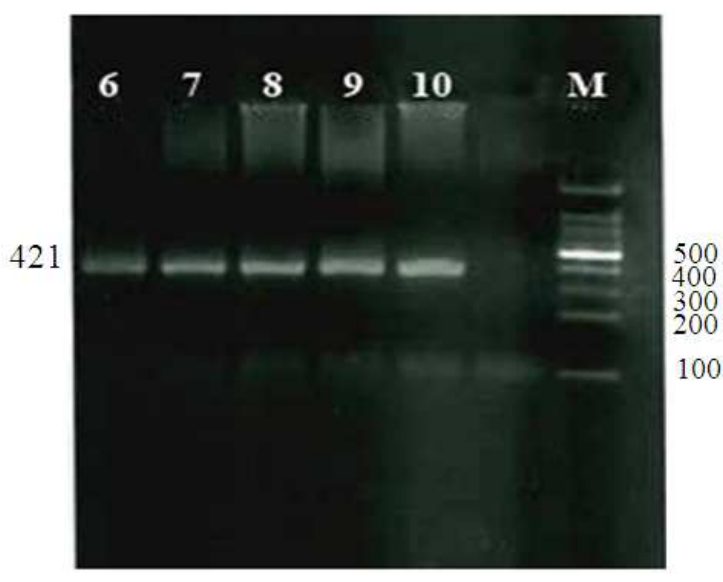

Fig. 6. Electrophoresis of the $18 \mathrm{~S}$ rRNA gene used for PCR assays for the local tested isolates from $S$. buffalonis. Lane M 100-bp ladder = DNA. Lanes 6-10 represent the positive diagnostic bands of the tested specimens

\subsection{Molecular Identification}

The revealed diagnostic DNA bands representing the amplified target gene $18 \mathrm{~S}$ rRNA appeared at 490 bp and 421 bp for $S$. fusiformis and S. buffalonis, respectively (Fig. 5 and 6 ).

The assembling of DNA sequences in both directions using forward and reverse primers yielded a fragment containing 421 consensus nucleotides for both sequences of the two isolates, $S$. fusiformis and $S$. buffalonis. Depending up on the BLAST comparative analysis, the partial 18S rRNA gene sequences of our local tested isolates of S. fusiformis and S. buffalonis showed (9\%) dissimilarity, (Fig. 7). The similarity percent of Egyptian strain of S.fusiformis with the published data of $S$. fusiformis under accession number U03071; (Holmdahl et al., 1994) was (99\%). On the other hand, the similarity between the present $S$. fusiformis and published data of $S$. fusiformis (accession numbers AF176926 and AF176927; (Yang et al., 2001b) was (97\%). Whereas, sequence dissimilarities between our isolate of $S$. fusiformis were $(7 \%)$ and $(8 \%)$ with $S$. buffalonis (accession number AF017121) and S. hirsuta (accession number AF017122), respectively.

Meanwhile, the sequence identity between the current isolate of $S$. buffalonis and published data of $S$. buffalonis (accession number AF017121) and S. hirsuta (accession number AF017122) were (98\%) and (97\%) respectively (Fig. 8). 


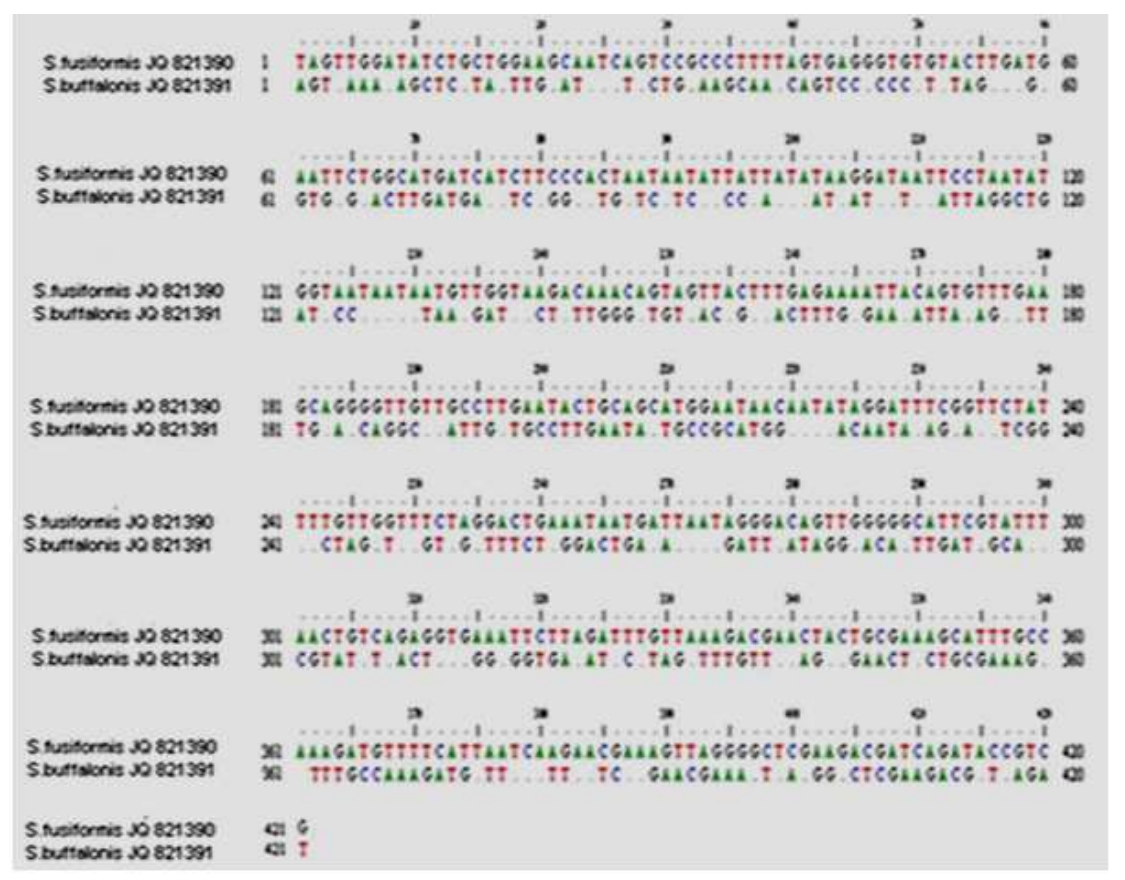

Fig. 7. Sequence alignments of the $18 \mathrm{~S}$ rRNA gene consisting of 421 nucleotides for each of the local tested isolates of $S$. fusiformis (GenBank accession number JQ821390) and S. buffalonis (GenBank accession number JQ821391) from Egyptian water buffaloes

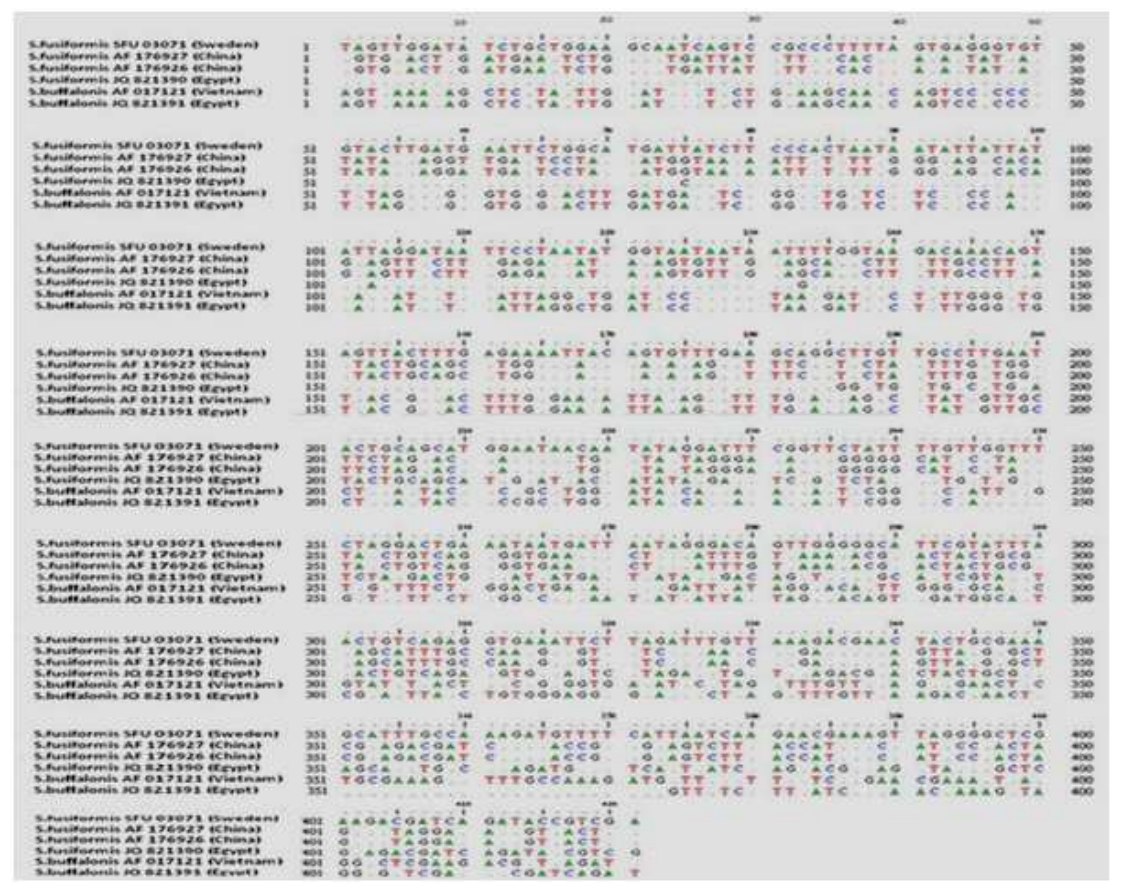

Fig. 8. Sequence alignment of $18 \mathrm{~S}$ rRNA gene for local tested isolates of both $S$. fusiforms and S. buffalonis compared with the published sequences of the same gene. The alignment was performed using MEGA version 4 


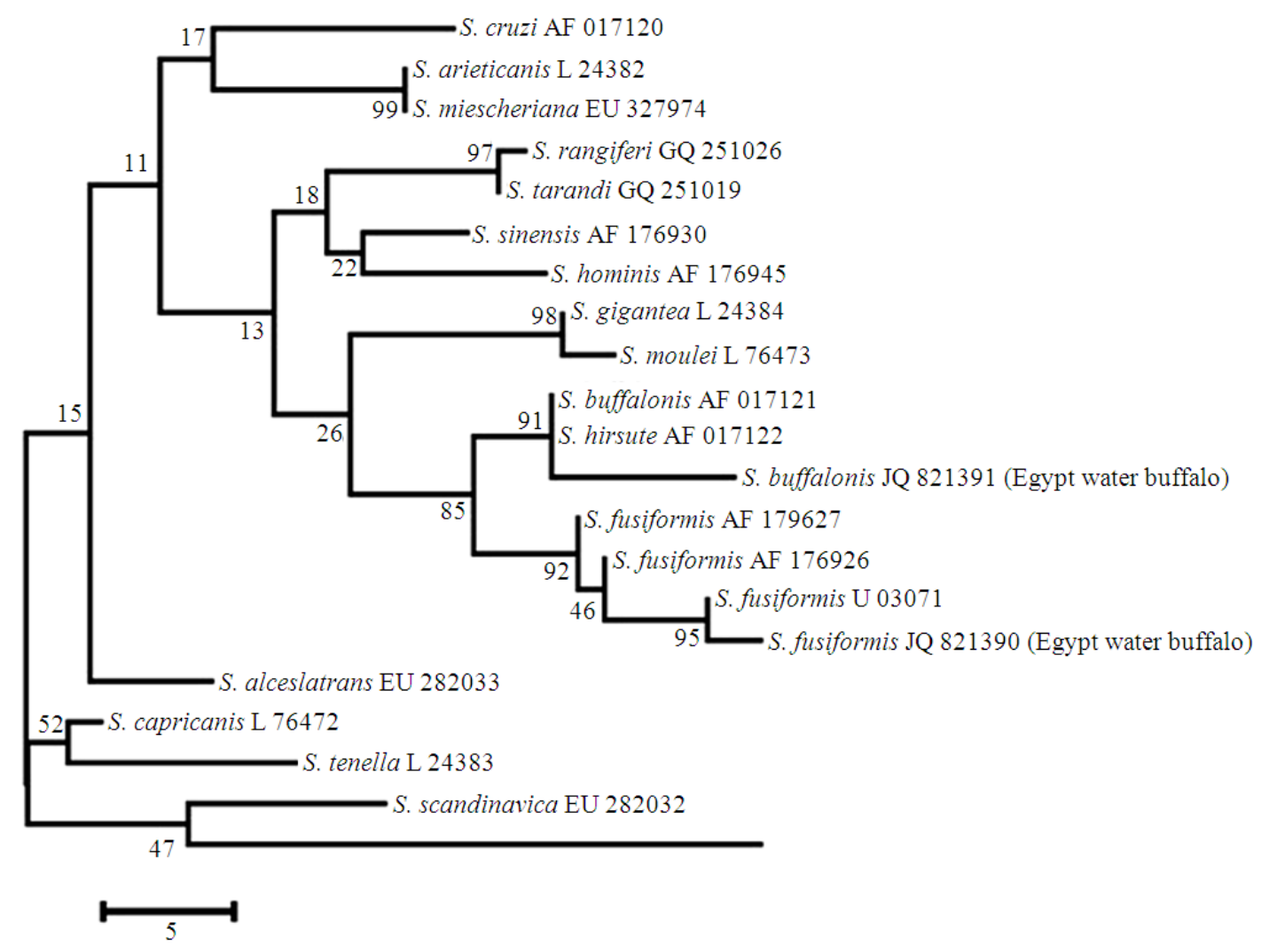

Fig. 9. Cladogram (phylogenetic tree) of Sarcocystis species based on the 18S rRNA gene sequence constructed according to maximum parsimony method. The branch lengths are proportional to the amounts of evolutionary changes. The phylogenetic tree indicates

The phylogenetic analysis of the $18 \mathrm{~S}$ rRNA sequences using N. caninum (GQ899206) as outgroup, showed that the Egyptian isolate of $S$. fusiformis placed in a group with the three previously reported $S$. fusiformis strains (accession numbers U03071, AF176926 and AF176927). Furthermore, the cladogram revealed that this group was more associated with the newly identified $S$. buffalonis isolates from the Egyptian water buffaloes (accession number JQ821391) together with $S$. buffalonis (AF017121) and S. hirsuta (AF017122) (Fig. 9).

\section{DISCUSSION}

Sarcocystosis is common within a wide host range of vertebrates as well as humans (Abdel-Ghaffar et al., 1978; 1990; Abdel-Ghaffar and Al-Johany, 2002).

Few decades ago, the prevalence of Sarcocystis species in Egyptian water buffaloes (Bublus bubalis) has been reported from different regions in Egypt, with few investigations studied detailed identification of the revealed Sarcocystis species. (Abdel-Ghaffar et al., 1978) examined the oesophagi of 20 Egyptian water buffaloes aged 7-10 years and found that all of them were infected with $S$. fusiformis. In addition, (Khalifa et al., 2008) examined specimens from tongue, heart, esophagus and skeletal muscles of 100 water buffaloes slaughtered in Sohag province, Egypt, revealing an infection rate of $28 \%$ with only S. fusiformis. El-Dakhly et al. (2011) examined 379 water buffaloes from the main slaughterhouses of BeniSuef province, Egypt and found that 299 were infected with overall prevalence of $78.9 \%$. Based on age, the examined animals were categorized into 3 groups. Infection rates with both macroscopic, S. fusiformis and microscopic, S. levinei, were $74.5,82.3$ and $81.2 \%$ in animals aged $1.5-2$ years, adult females aged 2-5 years and females aged over 5 years, respectively. Moreover, (Hilali et al., 2011) recorded, for the first time, the infection of water buffaloes in Egypt with the microscopic S. dubeyi species with a prevalence rate of $30 \%$. 
Mahmoud El-Seify et al. / American Journal of Animal and Veterinary Sciences 9 (2): 95-104, 2014

Table 1. Prevalence rates with S. fusiformis and S. buffalonis among the two groups of Egyptian water buffaloes (Bubalus bubalis) relative to age during the period extending from December 2011 to November 2012

\begin{tabular}{|c|c|c|c|c|c|c|c|}
\hline \multirow[b]{2}{*}{ Age groups } & \multirow{2}{*}{$\begin{array}{l}\text { Total numbers of } \\
\text { examined animals }\end{array}$} & \multicolumn{2}{|c|}{$\begin{array}{l}\text { Animals infected with } \\
\text { S. fusiformis }\end{array}$} & \multicolumn{2}{|c|}{$\begin{array}{l}\text { Animals infected with } \\
\text { S. buffalonis }\end{array}$} & \multicolumn{2}{|c|}{$\begin{array}{l}\text { Animals with mixed } \\
\text { infection }\end{array}$} \\
\hline & & No & $\%$ & No & $\%$ & $\mathrm{~N}$ & $\%$ \\
\hline $2-3$ years & 157 & 27 & 17.2 & 16 & 10.2 & 47 & 30 \\
\hline 5 years and above & 371 & 253 & 68.2 & 49 & 13.2 & 311 & 84 \\
\hline
\end{tabular}

In the current study, infection rates with $S$. fusiformis were $68.2 \%$ in old-aged water buffaloes (5 years and above) and $17.2 \%$ in younger age group (2-3 years). The rate of infection with $S$. buffalonis in old-aged animals was $13.2 \%$, while it was $10.2 \%$ in younger age group. Mixed infection was observed in $83.8 \%$ of the old-aged group, while it was $29.9 \%$ in younger animals (Table 1).

The present investigation revealed the occurrence of S. fusiformis and S. buffalonis in water buffaloes in Egypt with the first molecular characterization of them in the country. However, both species were reported from water buffaloes worldwide including China ( $\mathrm{Li}$ et al., 2002; Yang et al., 2001b). Vietnam (Jehle et al., 2009) and Iran (Oryan et al., 2011). Huong (1999a; 1999b) reported four species Sarcocystis, S. levinei, S. dubeyi, S. buffalonis and $S$. fusiformis in Vietnam. Moreover, (Claveria and Cruz, 2000) showed S. levinei in Philippines' water buffaloes.

Depending on the main fact that the morphometric characteristics of the cyst wall are the basic criteria for identification of different Sarcocystis species within the same intermediate host (Dubey et al., 1989; Xiang et al., 2010; El-Morsey et al., 2014). Moreover, Xiang et al, (2010) stated that light microscopy might be used to demonstrate the occurrence of sarcocysts in mammalian tissues and could be employed to discriminate among some parasite species based on the cyst wall structure as well as the size of the sarcocysts. Initially, the present work reported that water buffaloes in Egypt were infected by another macroscopic species, namely $S$. buffalonis, which was first previously recorded in Vietnam by (Huong et al., 1997). In the current investigation, cysts of $S$. buffalonis measured 1-3×0.1$0.5 \mathrm{~mm}$ and were comparatively smaller than those previously described by (Huong et al., 1997) (1-8×0.1$0.5 \mathrm{~mm}$ ) in Vietnam. Furthermore, S. buffalonis cysts, described herein and were surrounded by striated thick cyst wall measured from 4-6 $\mu \mathrm{m}$, while (Huong et al., 1997) reported that the cyst wall measured 3-7.7 $\mu \mathrm{m}$ thick.

S. buffalonis sarcocysts measured 1-3 mm long and appeared thread-like under the perimysial connective tissue along the longitudinal axis of the muscle fibers.
Some specimens appeared twisting as a result of the postmortem contraction of the muscle fibers.

With light microscopy, the cyst wall of S. buffalonis appeared thick, measuring 4-6 $\mu \mathrm{m}$ and characterized by the presence of striations (palisade-like). On the other hand, S. fusiformis cysts had thin and smooth wall that measured 1-3 $\mu \mathrm{m}$. S. buffalonis cysts were only detected in the oesophageal muscles (skeletal muscles were not included). Huong et al. (1997) found them in skeletal muscles, mainly abdominal muscles (49\%), cervical muscles (44\%), esophagus (27\%) and tongue (25\%) with the heart was free. Huong (1999a) reported that $S$. buffalonis were present in cervical muscles (14.3\%), abdominal muscles (16\%), tongue $(8 \%)$ and no infected cases in the heart. On the other hand, (Oryan et al., 2010) conducted the distribution pattern of Sarcocystis species in slaughtered water buffaloes (Bubalus bubalis) in Khuzestan, Iran by macroscopic and histological examination. They investigated esophagus, heart, diaphragm, tongue, masseter and thigh muscles. Among 100 examined water buffaloes, three $(3 \%)$ cases had infection with macroscopic Sarcocystis in the esophagus and thigh muscles, while $83 \%$ (83/100) revealed microscopic cysts. The highest prevalence of microscopic cysts was found in masseter muscle $(57.1 \%)$, followed by tongue, diaphragm, esophagus, heart and finally, thigh muscles $(30.0 \%)$. There was no significant difference between males $(83.6 \%)$ and females $(82.0 \%)$. They relayed on the size of the sarcocysts, wall thickness and location of cysts, as basis for species identification. Macroscopic, S. buffalonis and microscopic forms, S. levinei and $S$. dubeyi, were found in examined muscles of water buffaloes, while $S$. fusiformis sarcocysts could not be detected in the internal organs of the examined water buffaloes.

Recently, the 18S rRNA genes have been extensively used as suitable targets for the accurate identification of the closely related species of Sarcocystis as well as phylogenetic analyses (Holmdahl et al., 1999; Li et al., 2002; Dahlgren and Gjerde, 2007; Jehle et al., 2009). The conserved regions of $18 \mathrm{~S}$ rRNA gene help in designing primers that can be used to amplify the same 
gene in related species. Its mosaic structure allows flexibility in experimental designs for various phylogenic studies (Olsen and Woese, 1993). Furthermore, within Sarcocystis species, the sequences of this gene have variable genotypic behaviors (Holmdahl et al., 1999). In addition, (Holmdahl et al., 1999) found that the $18 \mathrm{~S}$ rRNA gene showed a high congruence of identity values within species, so it is a valuable to identify Sarcocystis species based on analysis of this gene.

The results of phylogenetic analysis revealed that there was a far relationship between the local tested isolates of both S. fusiformis and S. buffalonis according to achieved sequences of $18 \mathrm{~S}$ rRNA gene and the phylogenic tree analysis (Fig. 9). Meanwhile, the cladogram indicated that our local tested samples of $S$. buffalonis (accession number JQ821391) was closely related to S. buffalonis, accession number AF017121 (Holmdahl et al., 1999) and this was reflected by the high nucleotide homology (98\%) between them. On the contrary, the relationship with the tested isolate of $S$. fusiformis (accession number JQ821390) was highly different and represented by $(9 \%)$ nucleotide dissimilarity, suggesting a distinct genetic difference between the tested isolates of $S$. buffalonis and $S$. fusiformis investigated in the current study. However, at the same time, all these Sarcocystis species are related to each other as they utilize water buffaloes and cats as intermediate and final hosts, respectively. Holmdahl et al. (1999) stated that most of the Sarcocystis species clearly differed from one another in more than 100 nucleotides positions with the exception for S. buffalonis and S. hirsuta (13 positional differences). Moreover, the genotypic similarity of these species was also reflected in their close phenotypic resemblance (both species are macroscopic, thread-like and have cats as final hosts despite $S$. buffalonis is a parasite of water buffalo and S. hirsuta is a parasite of cattle). Yang et al. (2001a) mentioned in their analysis that the minimum relative sequence diversity between S. fusiformis and S. hirsuta was $1.7 \%$. Significant macroscopic, histological differences as well as data of sequence and phylogenetic analyses suggest a high genetic variability between both local tested isolates of S. fusiformis and S. buffalonis.

In the present study, phylogenetic analyses revealed that sequences of the local tested isolates of $S$. fusiformis, S. buffalonis, S. buffalonis (AF017121) and S. hirsuta (AF017122) shared the same definitive host (cat) and formed one cluster.

The 18S rRNA gene is characterized by the presences of various loci of polymorphism between the different species of Sarcocystis and within same species (Holmdahl et al., 1999; Oryan et al., 2011) this was observed in the current study and indicated by differences in the nucleotide sequences from the same Sarcocystis sp. Accordingly, the present Egyptian S. fusiformis differed by (3\%) and (1\%) from those previously recorded from China and Sweden, respectively (Yang et al., 2001b; Holmdahl et al., 1994). Therefore, examination of more sarcocysts from other geographic locations and sequencing at more genetic loci (for example, 28S rRNA and ITS-1) may elucidate whether there are more differences within the same species. Another possibility of nucleotide variability within the same Sarcocystis species is that, water buffaloes in different geographic regions may harbor different strains of the parasite. Moreover, (Oryan et al., 2011) revealed that the $18 \mathrm{~S}$ rRNA gene possesses different genetic variants which may arise as a result of dissimilarities among the multiple copies of this gene that were amplified from different merozoites within the sarcocyst.

\section{CONCLUSION}

Higher infection rates with both macroscopic $S$. fusiformis and S. buffalonis spp. within older age water buffaloes were observed. This may be due to that the sarcocysts need longer time to appear macroscopically. Economic losses may occur as a result of condemnation of carcasses infected with macroscopic sarcocysts in addition to infection with some pathogenic species of the sarcocyst cause weight gain, poor feeding efficiency, anorexia, fever, anemia, muscle weakness, reduced milk yield, abortion and mortality of intermediate hosts. To the best knowledge of the authors, the present work included the first molecular identification of $S$. fusiformis in Egypt, together with the first record of infection of Egyptian water buffaloes with $S$. buffalonis, depending up on both the morphological characteristics of the two species and the molecular differences between the two isolates as deduced from the analyses of $18 \mathrm{~S}$ rRNA gene sequences. However, further investigations on other genetic markers such as $28 \mathrm{~S}$ rRNA and ITS1 genes, are required for complete differentiation between currently detected Sarcocystis spp and the formerly identified species those were recorded from various geographic localities. Strict control measures should be applied on stray dogs and cats in developing countries especially Egypt, as they play serious roles in transmitting infection with different species of Sarcocystis to water buffaloes. 


\section{REFERENCES}

Abdel-Ghaffar, F. and A.M. Al-Johany, 2002. A light and electron microscope study of Sarcocystis mitrani sp. Nov. infecting the skink Scincus mitranus in the central region of Saudi Arabia. Parasitol. Res., 88: 102-106. DOI: 10.1007/s004360100506.

Abdel-Ghaffar, F., A.R. Bashtar, M.B. Ashour and T.H. Sakran, 1990. Life cycle of Sarcocystis gongyli Trinci 1911 in the shink Chalcides ocellatus and the snake Spalerosophis diadema. Parasitol. Res., 76: 444-450. DOI: 10.1007/BF00933555

Abdel-Ghaffar, F., M. Hilali and E. Scholtyseck, 1978. Ultrastructure study of Sarcocystis fusiformis (Railliet 1897) infecting the Indian water buffalo Bubalus bubalis of Egypt. Tropenmed. Parasitol., 29: 289-294. PMID: 103263.

Claveria, F.G. and M.J. Cruz, 2000. Sarcocystis levinei infection in Philippine water buffaloes (Bubalus bubalis). Parasitol. Int., 48: 243-247. DOI: 10.1016/S1383-5769(99)00025-2

Dahlgren, S. and B. Gjerde, 2007. Genetic characterization of six Sarcocystis species from reindeer (Rangifer tarandus tarandus) in Norway based on the small subunit rRNA gene. Vet. Parasitol., 146: 204-213. DOI: 10.1016/j.vetpar.2007.02.023

Dubey, J.P., C.A. Speer and R. Fayer, 1989. Sarcocystosis in Animals and Man, 1st Edn., CRC, Boca Raton, Florida, pp: 1-145.

El-Dakhly, K.H.M., K.H.A. El-Nesr, E. El-Nahass, A. Hirata and T. Yanai et al., 2011. Prevalence and distribution patterns of Sarcocystis spp. In buffaloes in Beni-Suef, Egypt. Trop. Anim. Health Prod., 43: 1549-1554. DOI: 10.1007/S11250-011-9840-2

El-Morsey, A., 2010. Studies on Sarcocystis species infecting water buffaloes in Egypt. M.D. Thesis Parasitology Dept. Fac. Of .Vet. Med. KafrElsheikh University.

El-Morsey, A., M. El-Seify, A.Y. Desouky, M.M. Abdel-Aziz and T. Yanai et al., 2014. Morphologic identification of a new Sarcocystis sp. In the common moorhen (Gallinula chloropus) (Aves: Gruiformes: Rallidae) from Brolos Lake, Egypt. Parasitol. Res., 113: 391-397. DOI: 10.1007/s00436013-3667-x
Hilali, M., M. El-Seify, A. Zayed, A. El-Morsey and J.P. Dubey et al., 2011. Sarcocystis dubeyi (Huong and Uggla, 1999) Infection in Water Buffaloes (Bubalus bubalis) from Egypt. J. Parasitol., 97: 527-528. DOI: 10.1645/GE-2656.1

Holmdahl, O.J., D.A. Morrison, J.T. Ellis and L.T. Huong, 1999. Evolution of ruminant Sarcocystis (Sporozoa) parasites based on small subunit rDNA sequences. Mol. Phylogenet. Evol., 11: 2737. PMID: 10082608

Holmdahl, O.J.M., J.G. Mattsson, A. Uggla and K.E. Johansson, 1994. The phylogeny of Neospora caninum and Toxoplasma gondii based on ribosomal RNA sequences. F.E.M.S. Microbiol. Lett., 119: 187-192.

Huong, L.T. and A. Uggla, 1999. Sarcocystis dubeyi n. sp. (Protozoa: Sarcocystidae) in the water buffalo (Bubalus bubalis). J. Parasitol., 85: 102104. PMID: 10207372

Huong, L.T.T., 1999a. Sarcocystis infections of the water buffalo in Vietnam. PhD Thesis, Swedish University of Agricultural Sciences, Uppsala.

Huong, L.T.T., 1999b. Prevalence of Sarcocystis spp. In water buffaloes in Vietnam. Vet. Parasitol., 86: 33-39. DOI: 10.1016/S0304-4017(99)00135-1

Huong, L.T.T., J.P. Dubey, T. Nikkilä and A. Uggla, 1997. Sarcocystis buffalonis n. sp. (Protozoa: Sarcocystidae) from the water buffalo (Bubalus bubalis) in Vietnam. J. Parasitol., 83: 471-474. PMID: 9194830

Jehle, C., A. Dinkel, A. Sander, M. Morent and T. Romig et al., 2009. Diagnosis of Sarcocystis spp. In cattle (Bos Taurus) and water buffalo (Bubalus bubalis) in Northern Vietnam. Vet. Parasitol., 166: 314-320. DOI: 10.1016/j.vetpar.2009.08.024

Khalifa, R.M., N.A. El-Nadi, F.G. Sayed and E.K. Omran, 2008. Comparative morphological studies on three Sarcocystis species in Sohag, Egypt. J. Egypt Soc. Parasitol., 38: 599-608. PMID: 18853631

Li, Q.Q., Z.Q. Yang, Y.X. Zuo, S.W. Attwood and X.W. Chen et al., 2002. A PCR-based RFLP analysis of Sarcocystis cruzi (Protozoa: Sarcocystidae) in Yunnan province, PR China, reveals the water buffalo (Bubalus bubalis) as a natural intermediate host. J. Parasitol., 88: 12591261. DOI: 10.1645/0022-3395(2002)088 
Odening, K., H.H. Wesemeier, G. Walter and I. Bockhardt, 1995. On the morphological diagnostics and host specificity of the Sarcocystis species of some domesticated and wild bovini (cattle, banteng and bison). Applic. Parasitol., 36:161-178. PMID: 8541889

Odening, K., M. Stolte and I. Bochardt, 1996. On the diagnostics of Sarcocystis in cattle: Sarcocystis of a species unusual for Bos taurus in a dwarf zebu. Vet. Parasitol., 66: 19-24. DOI: 10.1016/S03044017(96)00993-4

Olsen, G.J. and C.R. Woese, 1993. Ribosomal RNA: A key to phylogeny. FASEB J., 1: 113-123. PMID: 8422957

Oryan, A., H. Sharifiyazdi, M. Khordadmehr and S. Larki, 2011. Characterization of Sarcocystis fusiformis based on sequencing and PCR-RFLP in water buffalo (Bubalus bubalis) in Iran. Parasitol. Res., 109: 1563-1570. DOI: 10.1007/s00436-011-2412-6

Oryan, A., N. Ahmadi and S.M. Mousavi, 2010. Prevalence, biology and distribution pattern of Sarcocystis infection in water buffalo (Bubalus bubalis) in Iran. Trop. Anim. Health Prod., 42: 1513-1518. DOI: 10.1007/S11250-010-9601-7

Tamura, K., J. Dudley, M. Nei and S. Kumar, 2007. MEGA4: Molecular Evolutionary Genetics Analysis (MEGA) software version 4.0. Mol. Bio. Evol., 24: 1596-1599. PMID: 17488738
Xiang, Z., B.M. Rosenthal, Y. He, W. Wang and H. Wang et al., 2010. Sarcocystis tupaia, sp. nov., a new parasite species employing treeshrews (Tupaiidae,Tupaia belangeri chinensis) as natural intermediate hosts. Parasitol. Int., 59: 128-132. DOI.10.1016/j.parint.2009.12.003

Yang, Z.Q., Y.X. Zuo, B. Ding, X.W. Chen and J. Luo et al., 2000. 18S rRNA gene of Sarcocystis hominis cyst from water buffalo and cattle. Zool. Res., 21: 133-138.

Yang, Z.Q., Y.X. Zuo, B. Ding, X.W. Chen and J. Luo et al., 2001a. Identification of Sarcocystis hominis-like (Protozoa: Sarcocystidae) cyst in water buffalo (Bubalus bubalis) based on $18 \mathrm{~S}$ rRNA gene sequences. J. Parasitol., 87: 934-937. DOI: $10.1645 / 0022-$ 3395(2001)087[0934:IOSHLP]2.0.CO;2

Yang, Z.Q., Y.X. Zuo, Y.G. Yao, X.W. Chen and G.C. Yang et al., 2001b. Analysis of the 18S rRNA genes of Sarcocystis species suggests that the morphologically similar organisms from cattle and water buffalo should be considered the same species. Mol. Biochem. Parasitol., 115: 283-288. DOI: 10.1016/S0166-6851(01)00283-3 